\title{
Potent antitumor effect of combination therapy with sub-optimal doses of Akt inhibitors and pomalidomide plus dexamethasone in multiple myeloma
}

\author{
SHIORI KINOSHITA, MASAKI RI, TAKASHI KANAMORI, SHO AOKI, \\ TAKASHI YOSHIDA, TOMOKO NARITA, HARUHITO TOTANI, ASAHI ITO, \\ SHIGERU KUSUMOTO, TAKASHI ISHIDA, HIROKAZU KOMATSU and SHINSUKE IIDA \\ Department of Hematology and Oncology, Nagoya City University Graduate School of \\ Medical Sciences, Nagoya, Aichi 467-8601, Japan
}

Received September 2, 2017; Accepted January 22, 2018

DOI: $10.3892 / \mathrm{ol} .2018 .8501$

\begin{abstract}
Afuresertib (AFU), a novel inhibitor of the serine/threonine kinase AKT, has clinical efficacy as a monotherapy against hematological malignancies and is expected to be used in combination with standard therapies for multiple myeloma (MM). To develop a more effective and less toxic combination of immunomodulatory drugs (IMiDs) for therapy, the antitumor effect of sub-optimal doses of AFU, pomalidomide plus dexamethasone (PD), and the AFU-PD combination on MM cells were examined in the present study. Two MM cell lines, XG-7 and U266, with low sensitivity to both PD and AFU monotherapies, were subjected to these combinations and analyzed. Although the cell lines showed a slight reduction in viability with the sub-optimal doses of each monotherapy, the combination of the treatments resulted in a reduction in cell viability and the progression of apoptosis. Co-treatment with sub-optimal doses of PD and AFU enhanced caspase activation and highly suppressed the expression of IKZF1 and IKZF3. In addition, this combination promoted the dephosphorylation and stabilization of 4EBP1, an inhibitor of eIF4E activation, which led to the impairment of eIF4E-mediated translational activity. Furthermore, AFU showed a sufficient inhibitory effect on the phosphorylation of FOXO1, a tumor suppressor, in monotherapy or in combination with PD, which may be attributable to the
\end{abstract}

Correspondence to: Dr Masaki Ri, Department of Hematology and Oncology, Nagoya City University Graduate School of Medical Sciences, 1 Kawasumi, Mizuho-chou, Mizuho-ku, Nagoya, Aichi 467-8601, Japan

E-mail: rrmasaki@ybb.ne.jp

Abbreviations: AFU, Afuresertib; MM, multiple myeloma; IMiDs, immunomodulatory drugs; PD, pomalidomide plus dexamethasone, PIs, proteasome inhibitors; LEN, lenalidomide; POM, pomalidomide; BOR, bortezomib; DEX, dexamethasone

Key words: Akt inhibitor, immunomodulatory drugs, combination therapy, multiple myeloma, afuresertib, pomalidomide, lenalidomide activation of FOXO1, the subsequent inhibition of tumor growth, and the induction of cell death. In conclusion, the combination therapy with sub-optimal doses of PD and AFU exhibited potent antitumor activity in MM cells and may provide a novel strategy for the treatment of patients who experienced intolerable toxicity or insufficient response during IMiD therapy.

\section{Introduction}

The treatment of multiple myeloma (MM) has been markedly altered by the clinical use of proteasome inhibitors (PIs) and immunomodulatory drugs (IMiDs). Two IMiDs, lenalidomide (LEN) and pomalidomide (POM), have been characterized with potent immunostimulatory action, such as co-stimulatory effects on T and NK cells (1); therefore, they are often tested in combination with monoclonal antibodies, such as elotuzumab (2), daratumumab (3), and others (4). In addition to the stimulatory effect on the immune system, the intracellular action of MM cells targeting an ubiquitin E3 ligase, cereblon $(1,5)$, followed by the degradation of the proliferative factors, IKZF1 and IKZF3, have been also recognized as the main effectors of anti-tumor activity of these agents (6-8). Recently, the combination therapy of PI and IMiDs, such as LEN plus bortezomib (BOR) and dexamethasone (DEX), was successfully introduced to the initial treatment of MM in both transplant eligible and non-eligible subjects $(9,10)$, and is considered an appropriate treatment for suitable patients, mainly including transplant eligible or fit patients without complications. However, the detailed mechanisms of the anti-tumor effect of this combination therapy are poorly understood. In addition, antagonistic activity of the combination of PI and IMiDs was reported (11), in which the LEN-induced degradation of IKZF1, which proceeded through the modification of cereblon, was inhibited by proteasome inhibition. Therefore, except for monoclonal antibodies, excellent treatment strategies for combination with IMiDs have not been fully developed.

The PI3K/Akt pathway is constitutively activated in MM cells, to maintain the signals of proliferation, anti-apoptosis, and drug resistance in MM cells (12). Therefore, several inhibitors that target this pathway have been attempted to 
develop the treatment of relapsed and refractory MM with a single treatment or in combination with other agents.

Among these agents, afuresertib (AFU), a novel serine/threonine kinase Akt inhibitor, has demonstrated single-agent clinical activity and synergistic anti-myeloma effects against MM cells in combination with BOR plus DEX therapy (13).

In the present study, we investigated a more effective and less toxic combination of IMiDs for therapy, and estimated the additional effect of an Akt inhibitor in the combination treatments of IMiDs plus DEX and examined the cytotoxicity of IMiDs plus DEX and/or AFU treatment, with both DEX and AFU at suboptimal doses, against MM cells. We identified an enhanced anti-myeloma effect for this combination therapy and attempted to elucidate the mechanism underlying this enhanced activity with a focus on the previously reported factors involved in the functional mechanism of IMiDs or Akt inhibitors.

\section{Materials and methods}

Cell lines and primary MM cells. The human MM cell lines, JJN-3 and SK-MM-1, were purchased from DSMZ, the German Resource Center for Biological Material (Braunshweig, Germany). U-266 was purchased from the American Type Culture Collection (Maanassa, VA, USA). KMS-11, NOP1, NCI-H929, and XG-7 were kindly provided by Kawasaki Medical University, Aichi Cancer Center, Oita University, and Dr. Bernard Klein, respectively. All cell lines were cultured in RPMI-1640 medium (Thermo Fisher Scientific, Inc., Waltham, MA, USA) supplemented with $10 \%$ fetal bovine serum, $100 \mathrm{U} / \mathrm{ml}$ penicillin and $100 \mathrm{U} / \mathrm{ml}$ streptomycin at $37^{\circ} \mathrm{C}$ in $5 \% \mathrm{CO}_{2}$ incubator. Bone marrow mononuclear cells (BMNCs) were isolated from three patients with MM. MM cells were purified from BMNCs by using anti-CD138 Micro Beads (Miltenyi Biotec, Bergisch Gladbach, Germany) as previously described (14). Two primary MM cell sources, with abundant numbers of cells, were selected for immunoblot analysis. All donors provided informed written consent prior to sampling in accordance with the Declaration of Helsinki and the present study was approved by the institutional ethics committees of Nagoya City University Graduate School of Medical Sciences.

Antibodies and reagents. POM and LEN were provided by Celgene Co., Ltd and also purchased from Santa Cruz Biotechnology (Dallas, TX). AFU (GSK2110183) was purchased from Selleck Chemicals (Houston, TX). The antibodies to cleaved caspase-3 (no. 9664), cleaved caspase- 8 (no. 9496), IKZF1 (no. 5443), p-Akt (no. 9271), Akt (no. 9272), IRF4 (no. 4948 and no. 4964), p-FoxO1/FoxO3 (no. 9464), 4E-BP-1 (no. 9452), P-4E-BP-1 (no. 9459), and eIF4E (no. 9742) were purchased from Cell Signaling Technology, Inc. (Danvers, MA, USA). The antibody to actin (sc-1616) was purchased from Santa Cruz Biotechnology, Inc. (Dallas, TX, USA) and the antibody to IKZF3 (NBP-16938) was purchased from Novus Biologicals (Littleton, CO, USA), The proteins were visualized by using goat anti-rabbit IgG-HRP (sc-2004) and goat anti-mouse IgG-HRP (sc-2005) (Santa Cruz Biotechnology, Inc.).

Cell proliferation assay. The proliferation of MM cell lines was assessed by using Cell Titer 96 Aqueous One Solution cell proliferation assay kits (Promega Corporation, Madison, WI, USA), as previously described (15). Cells were seeded in 96-well flat bottom microplates $\left(2 \times 10^{4}\right.$ cells/well), and then treated with appropriate concentration of each agent in $5 \% \mathrm{CO}_{2}$ incubator at $37^{\circ} \mathrm{C}$ for $72 \mathrm{~h}$. Cell proliferation was measured at the indicated concentrations relative to the control. The absorbance at $490 \mathrm{~nm}$ was read using a 96-well plate reader. All expressed values represent the average of triplicate experiments and the $\mathrm{IC}_{50}$ values were computed by GraphPad Prism 6 (GraphPad Software, Inc., La Jolla, CA, USA) in accordance with the manufacturer's instructions.

Apoptosis assay. The apoptosis of MM cell lines was evaluated by using an Annexin V-FITC Apoptosis Detection Kit I (BD Biosciences, San Jose, CA, USA) in accordance with the manufacturer's instructions. the cells were treated with appropriate concentration of each agents for 48 or $72 \mathrm{~h}$ and reacted with FITC-conjugated Annexin V and PI for $15 \mathrm{~min}$ at room temperature in the dark. The cells were analyzed on a FACS Calibur flow cytometer (BD Biosciences) with the aid of Flow Jo software (Tree Star, Ashland, OR, USA). In the current study, we measured the ratio of Annexin $\mathrm{V}$ positive cells, generally including early and late apoptotic cells. We defined induced apoptotic cells as Annexin V positive cells measured at each treatment, and spontaneous apoptotic cells as Annexin V positive cells measured under the no treatment. The percentage of specific apoptosis (\% $\%$ specific apoptosis) was calculated as follows: 100x (\% induced apoptotic cells- $\%$ spontaneous apoptotic cells)/(100-\% spontaneous apoptotic cells). All expressed values represent the mean value of triplicate experiments.

Immunoblot analysis. The MM cell lines and primary tumor cells from patients with MM were incubated in the presence or absence of reagents for the indicated times. The cells were lysed in lysis buffer [20 mM HEPES ( $\mathrm{pH} 7.5$ ), 0.1\% NP40, $150 \mathrm{mM} \mathrm{NaCl}, 1 \mathrm{mM}$ EDTA (PH 8.0), 1 tablet of phosphatase inhibitor cocktail (Roche, Basel, Switzerland), 1 tablet of protease inhibitor cocktail (Roche)] at $4^{\circ} \mathrm{C}$ with sonication. The preparation and analyses of whole-cell extracts and were performed as previously described (15). After the estimation of the total protein content by using Bradford reagent (Sigma-Aldrich; Merck KGaA, Darmstadt, Germany), each loaded sample was adjusted to $30 \mu \mathrm{g}$ per $10 \mu \mathrm{l}$. The lysates were separated electrophoretically by SDS-PAGE (4-15\%) (Bio-Rad Laboratories, Hercules, CA, USA) and transferred to polyvinylidene difuroide membrane (Thermo Fisher Scientific, Inc.) using dry blotting system (Thermo Fisher Scientific, Inc.). The membranes were blocked with $2 \%$ skim milk for $1 \mathrm{~h}$ at room temperature and incubated with appropriate primary antibodies (dilution; 1:1,000 except for actin 1:2,000) at $4^{\circ} \mathrm{C}$ overnight. The membranes were incubated with secondary antibody (anti-rabbit; 1:10,000, anti-goat; 1:5,000) for $1 \mathrm{~h}$ at room temperature. The bound antibodies were detected by the enhanced chemiluminescence reagent (GE healthcare, Chicago, IL, USA). The results of the immunoblot analysis presented are representative of multiple experiments.

Statistical analysis. The statistical significance of differences among more three groups was evaluated by using ANOVA followed by post hoc Tukey test., computed using GraphPad 

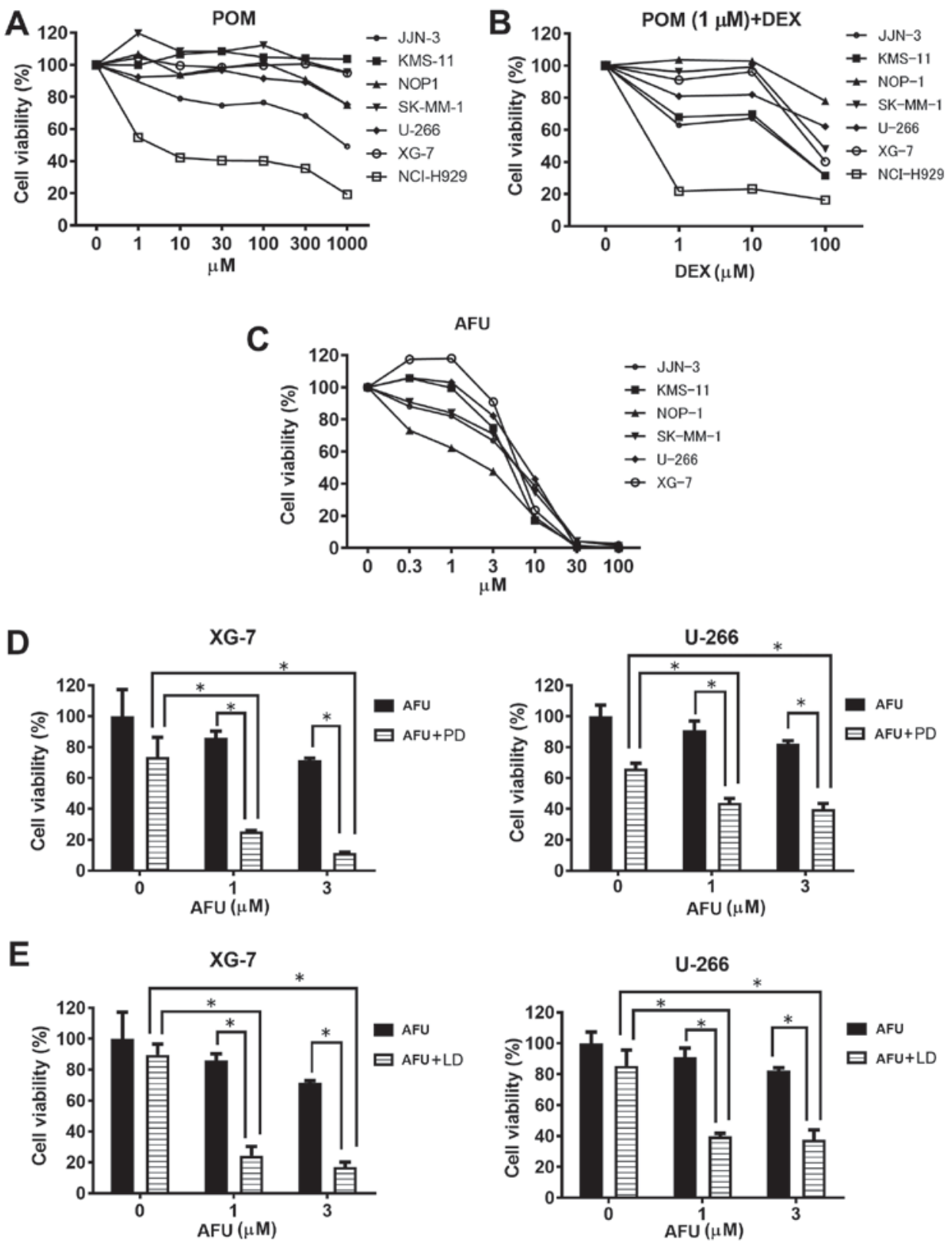

Figure 1. Growth inhibition of MM cells by pomalidomide, $1 \mu \mathrm{M}$ pomalidomide plus dexamethasone, and afuresertib. (A-C) The growth inhibitory effect of three therapies (pomalidomide; $1 \mu \mathrm{M}$ pomalidomide plus dexamethasone; and afuresertib) after treatment for $72 \mathrm{~h}$ in $\mathrm{MM}$ cells. The data represent the mean of three independent experiments. (D and E) Growth inhibitory effect of low-dose AFU treatment in combination with a suboptimal dose of pomalidomide $(1 \mu \mathrm{M})$ or lenalidomide $(1 \mu \mathrm{M})$ with dexamethasone on two MM cell lines. "Represents statistically significant $(\mathrm{P}<0.05)$, calculated by post hoc Tukey test. LD, lenalidomide plus dexamethasone; AFU, afuresertib; POM, pomalidomide; DEX, dexamethasone.

Prism 6 (GraphPad Software, Inc.). $\mathrm{P}<0.05$ was considered to indicate a statistically significant difference.

\section{Results}

Inhibitory activity of pomalidomide alone or in combination with dexamethasone against MM cell growth. First, we determined the suboptimal doses of each treatment, POM plus DEX (PD) and AFU, for MM cells before the examination of the AFU-PD combination. As shown in Fig. 1A, treatment with POM alone caused only a small inhibition of MM cell viability at all concentrations, except in NCI-H929, which is known to have a high sensitivity to IMiDs (6). We determined the suboptimal dose of POM as $1 \mu \mathrm{M}$, and then evaluated the effect of combination with $1 \mu \mathrm{M}$ POM and the indicated doses of DEX in MM cells (Fig. 1B). The PD combination with $100 \mu \mathrm{M}$ DEX markedly inhibited the vitality of most MM cell lines, but only moderate inhibition occurred with $10 \mu \mathrm{M}$ DEX. From these results, we determined the suboptimal dose of the PD treatment as $1 \mu \mathrm{M}$ POM plus $10 \mu \mathrm{M}$ DEX.

As shown in Fig. 1C, the monotherapy of AFU caused a dose-dependent inhibition of MM cell viability. A remarkable inhibition of viability was observed above $10 \mu \mathrm{M}$ AFU in most cell lines; therefore, concentrations of 1 and $3 \mu \mathrm{M}$ were concluded as the suboptimal doses of AFU treatment. Among the six MM cell lines tested, XG-7 and U266 showed comparatively lower sensitivity to AFU treatment. 
A Annexin V

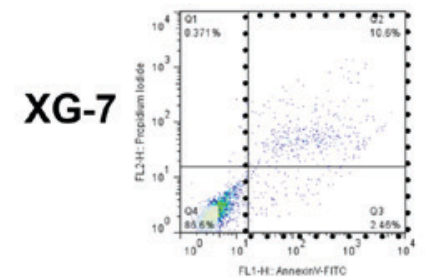

$13.1 \%$

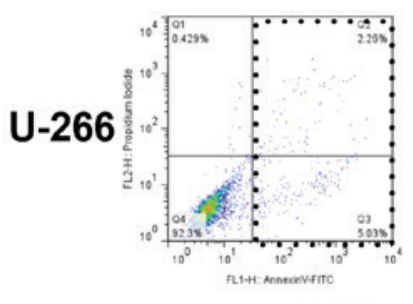

$7.3 \%$

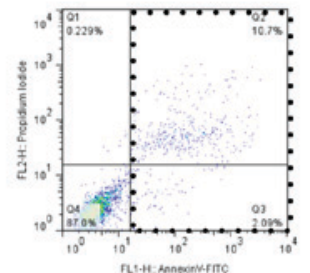

$12.8 \%$

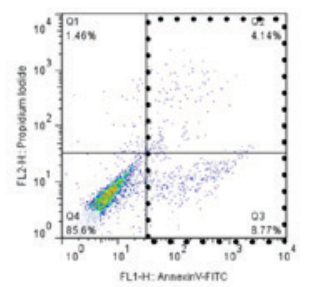

$12.9 \%$

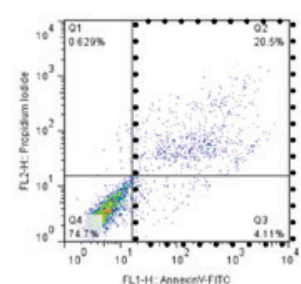

$24.6 \%$

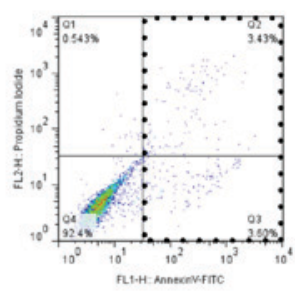

$7.0 \%$

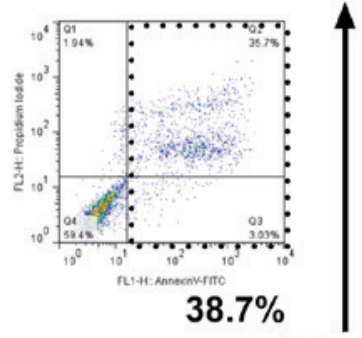

$38.7 \%$

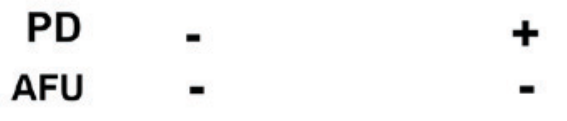

B
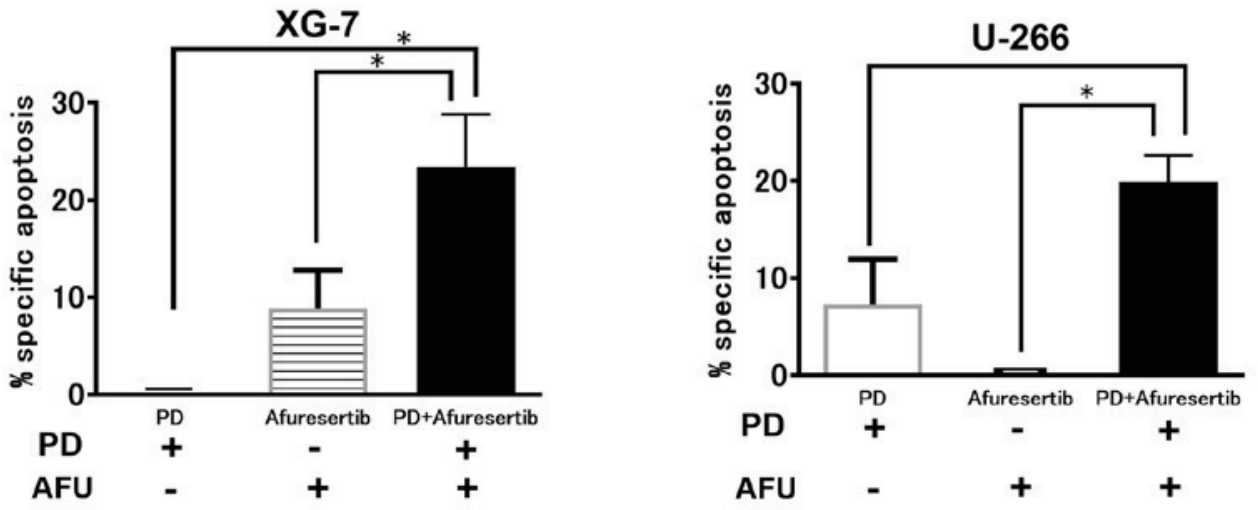

Figure 2. Induction of cell death by suboptimal dose of pomalidomide plus dexamethasone (PD), or afuresertib, and the afuresertib-PD combination in MM cells. (A) The evaluation of cell death by using Annexin V and PI staining in two MM cell lines treated with suboptimal doses of PD, or AFU, and their combination. AFU and PD are used as $1 \mu \mathrm{M}$ of AFU or $1 \mu \mathrm{M}$ of pomalidomide with $10 \mu \mathrm{M}$ of dexamethasone, respectively. XG-7 and U266 were subjected to the indicated treatments for 48 and $72 \mathrm{~h}$, respectively. A representative case of three independent experiments was shown. (B) The evaluation of the percentage of specific apoptosis in two cell lines treated with PD and or AFU. The mean value with SD bar of three independent experiments was shown. *Represents statistically significant $(\mathrm{P}<0.05)$, calculated by post hoc Tukey test. PD, pomalidomide plus dexamethasone; AFU, Afuresertib; MM, multiple myeloma.

Inhibition activity of IMiDs plus dexamethasone treatment in combination with AFU against MM cell growth. Two MM cell lines, XG-7 and U266, characterized by a comparatively lower sensitivity to AFU, were treated with the AFU-PD combination therapy. At the suboptimal doses of PD treatment, XG-7 showed a mild reduction $(28 \%)$ in viability in comparison with the untreated cells. Similarly, AFU treatment alone at the suboptimal dose showed a weak reduction in cell viability at the indicated concentrations: $10 \%$ at $1 \mu \mathrm{M}$ and $25 \%$ at $3 \mu \mathrm{M}$ (Fig. 1D, left). However, co-treatment with suboptimal doses of PD and AFU resulted in an enhanced reduction of cell viability: $75 \%$ at $1 \mu \mathrm{M}$ AFU and $85 \%$ at $3 \mu \mathrm{M}$ AFU. These reductions were superior to those observed with $\mathrm{PD}$ or AFU alone. The enhanced inhibition of cell viability caused by the combination was also shown in similar tests in U266 cells (Fig. 1D, right).
Next, we examined the effect of the combination therapy with LEN, instead of POM. Although $1 \mu \mathrm{M}$ of LEN plus $10 \mu \mathrm{M}$ of DEX treatment showed a lower reduction of viability (10\%) in XG-7 cells, the combination with AFU considerably reduced the viability at the indicated doses: $75 \%$ at $1 \mu \mathrm{M}$ of AFU and $80 \%$ at $3 \mu \mathrm{M}$ of AFU (Fig. 1E left), which were very similar to the results obtained in U266 cells with identical combination therapies (Fig. 1E right).

Evaluation of apoptosis in MM cells treated with the combination therapy of POM plus DEX and AFU treatment. As shown in Fig. 2A, PD or AFU treatment at a suboptimal dose triggered less apoptosis. However, the combination therapy augmented the progression of apoptosis in both XG-7 and U266 cells. The \%specific apoptosis in XG-7 cells treated with PD, AFU, or the AFU-PD combination was $0,13.5$, and 30.0 , respectively 


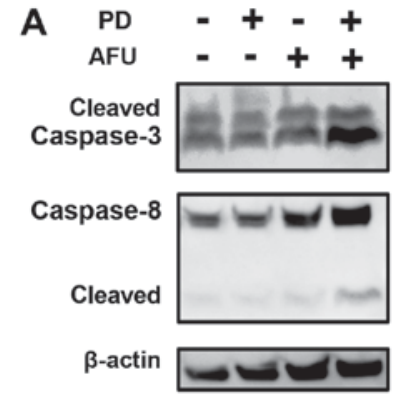

XG-7
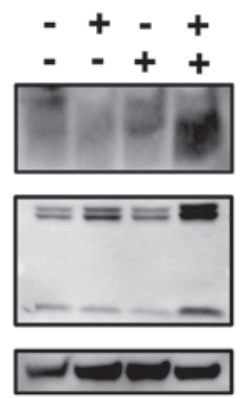

U-266
B $\mathrm{PD}$ - +

AFU

IKZF1

IKZF3

CRBN

\section{IRF4}

$\beta$-actin

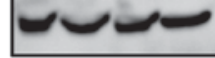

XG-7

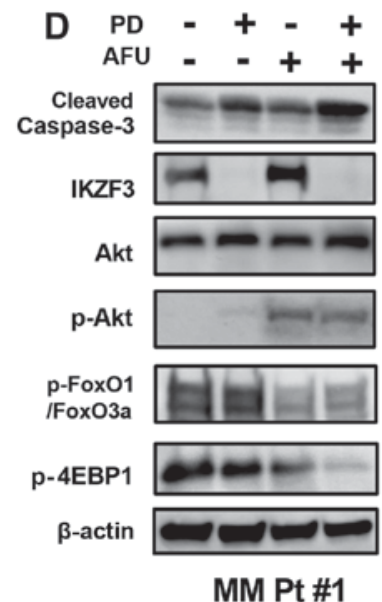

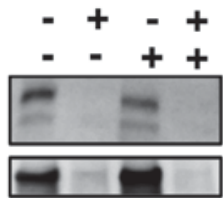
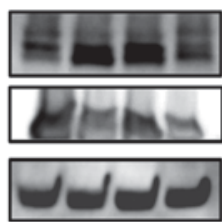

U-266
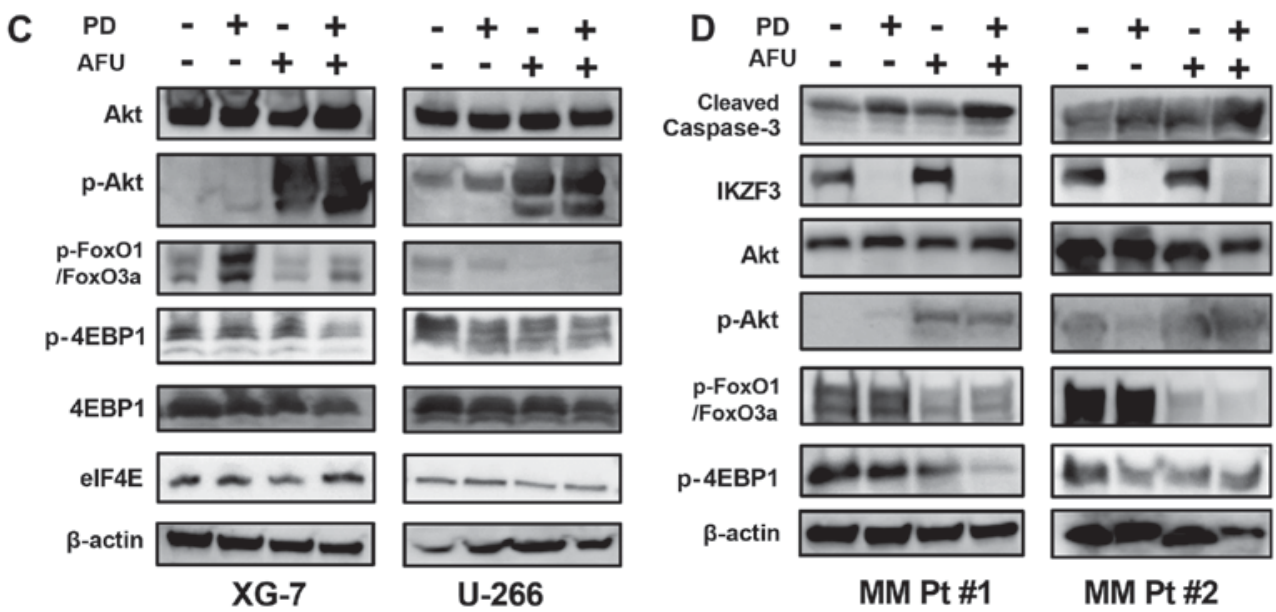

Figure 3. Analysis of the mechanism of action of the enhanced anti-tumor effect of the combination therapy of suboptimal doses of pomalidomide plus dexamethasone and afuresertib in MM cells. (A-C) The altered expression of protein substrates was analyzed in two MM cell lines that were treated with suboptimal doses of PD, or AFU, or the PD and AFU combination. The XG-7 and U266 cell lines were subjected to the indicated treatments for 48 and $72 \mathrm{~h}$, respectively. (A) Caspases, (B) substrates related mainly to the working mechanism of IMiDs, (C) substrates mainly related to the working mechanism of afuresertib, and (D) two primary MM cell cultures, were subjected to the analysis in the same manner as the two MM cell lines. In the expression panel of p-FoxO3a/FoxO1, the upper band represents p-FoxO3a and the lower band represents p-FOXO1. PD, pomalidomide plus dexamethasone; AFU, Afuresertib; MM, multiple myeloma; IMiDs, immunomodulatory drugs.

(Fig. 2B, left). Similar results were also observed in U266 cells; the \%specific apoptosis induced by treatment with PD, AFU, or the AFU-PD combination was 5.0,0, and 22.0, respectively (Fig. 2B, right).

Alteration of IKZF- and Akt-regulating substrates in MM cells after co-treatment with suboptimal doses of POM plus DEX and/or AFU. The AFU-PD combination therapy with suboptimal doses of PD and AFU enhanced caspase activation, as indicated by the overexpression of cleaved caspase- 3 and -8 , in XG-7 cells in comparison with the individual treatments. Similar results were also observed in U266 cells treated in an identical manner (Fig. 3A). PD treatment suppressed the expression of IKZF1 and IKZF3 in both XG-7 and U266 cells, even at suboptimal doses, which did not occur after the treatment with AFU alone. In the AFU-PD combination therapy with suboptimal doses of PD and AFU, the suppression of both IKZF1 and IKZF3 was further enhanced in XG-7 cells. In U266 cells, the PD-induced suppression of IKZF1 and IKZF3 was maintained after the addition of AFU. The accumulation of cereblon, observed in PD treatment, was also maintained after the co-treatment with AFU in both MM cell lines. The expression of IRF4 was decreased by the PD treatment, and this reduction was sustained when combined with AFU treatment in both cell lines (Fig. 3B).
In agreement with other reports (16), AFU treatment triggered a feedback increase in Akt phosphorylation in both MM cell lines, which reflected the strong inhibition of kinase activity in Akt. Although this feedback increase was not induced in PD treatment, it was observed in the AFU-PD combination treatment with suboptimal doses of AFU and PD.

FOXO1, which is considered a tumor suppressor gene regulated by Akt signaling (17), was highly phosphorylated in both MM cell lines. After PD treatment, the level of phosphorylation was increased in XG-7 cells and was slightly suppressed in U266 cells. Following the AFU-PD combination treatment administered at suboptimal doses of each agent, the upregulation of phosphorylated FOXO1 was inhibited in XG-7 cells, and its expression was profoundly suppressed in U266 cells.

4EBP1, a negative regulator of eIF4e (18), was phosphorylated in both MM cell lines (Fig. 3C). Moderate dephosphorylation of 4EBP1 was observed in PD-treated U266 cells, but not in XG-7 cells. However, the AFU-PD combination at suboptimal doses of AFU and PD triggered the dephosphorylation of 4EBP1 in both MM cell lines.

Two primary MM cells derived from the patients were treated with PD, AFU, and AFU-PD subjected to western blotting analysis (Fig. 3D). In the PD and AFU combination treatment, the enhanced activation of caspase-3, 
dephosphorylation of FOXO1 and 4EBP1, and repression of IKZF3 were observed in all primary MM cells.

\section{Discussion}

AFU, a member of a family of ATP-competitive inhibitors, mainly targets the ATP binding and subsequent phosphorylation of Akt substrates; it does not affect the localization and self-activation of Akt. In previous reports, the hyper-phosphorylation of Akt was commonly observed with other ATP-competitive type inhibitors, such as A-4436548 (19) and GSK690693 (20), and a similar hyper-phosphorylation of Akt was also observed in malignant pleural mesothelioma cells treated with AFU (21). Although the precise mechanism of this hyper-phosphorylation is not well understood, it may be considered as the reflection of a homeostatic feedback mechanism by which the cell attempts to maintain Akt activity during the inhibition of phosphorylation of Akt substrates caused by ATP-competitive inhibitors (19).

In general, most ATP-competitive kinase inhibitors have been reported to inhibit additional kinases, such as PKA and PKG1 $\alpha$, through off-target action (16); therefore, it cannot be completely excluded that off-target action may be associated with the anti-tumor effect of AFU on MM cells. In our study, Akt treatment was tested at a sub-optimal dosage, which would serve to minimize the effect of potential off-target action on the anti-tumor effect of AFU on MM cells.

Several studies have reported the mechanism of action responsible for the anti-tumor activity of IMiDs. Among them, we have focused on two particular activities and evaluated the alteration of factors related to the two activities that follow the addition of a suboptimal dose of AFU to PD treatment. One of these activities was the regulation of eIF4E, which resulted in the inactivation of C/EBP translation and the subsequent impairment of IRF4 transcription (18). The second was the modulation of CRBN specificity for the ubiquitination of proteins, which led to the downregulation of IKZF substrates (8). Among the factors related to the above activities, we focused mainly on two factors observed in the combination treatment with AFU and PD: The dephosphorylation of 4EBP associated with the inactivation of eIF4E-related transcriptional activity (22) and the altered phosphorylation level in FOXO1, a proapoptotic factor associated with cell cycle arrest and cell death (23-26).

A previous report demonstrated that $10 \mu \mathrm{M}$ of IMiD compounds downregulated eIF4E expression at the mRNA level, which impaired C/EBP translation and inhibited MM cell growth (18). In this previous report, MM cells with IMiD resistance did not show suppressed eIF4E expression and sustained C/EBP translation during IMiD treatment. The suboptimal dose, $1 \mu \mathrm{M}$ of POM treatment could not reduce eIF4E expression in MM cells in the present study. However, when a lower dose of an Akt inhibitor was added, 4EBP1, a regulator of eIF4E and ordinarily inactivated by the phosphorylation (22), was dephosphorylated and activated. Therefore, it is speculated that this alternative activity of 4EBP1 may inhibit the activation of eIF4E through the impairment of translation activity without the suppression of eIF4E expression at the protein level, which leads to further suppression of IRF4. A similar impairment of eIF4E was also observed by
mTOR inhibitors in combination with IMiDs $(18,27)$. These results suggested that the inactivation of the eIF4E-related translational complex by Akt or mTOR inhibition can be an effective strategy for the treatment of relapsed or refractory MM (RRMM) that was insensitive or resistant to IMiDs treatment owing to the poor suppression of eIF4E expression during IMiDs treatment.

FOXO1 is reported as a tumor suppressor gene, but may act as a pro-apoptotic factor (23-26). Many tumors have phosphorylated Akt, which inhibits FOXO1 activity by direct phosphorylation and cytoplasmic sequestration, and contributes to the maintenance of tumor cell survival. In the present study, MM cells had highly phosphorylated FOXO1 proteins that were regulated by Akt activation. In the current study, we could not determine a general evaluation of the effect of PD treatment on the phosphorylation level of FOXO1 in MM cells. Regardless of the changes in FOXO1 phosphorylation caused by PD treatment, the addition of AFU showed sufficient inhibition to prevent the accelerated phosphorylation of FOXO1 in an MM cell line, or repress the constant phosphorylation of FOXO1 in two primary samples. These results suggest that AFU exerted a sufficient inhibitory effect on FOXO1 phosphorylation, regardless of the effect of PD therapy, in combination with PD. Therefore, additional AFU treatment would be the preferred strategy to improve the sensitivity to IMiD activity and overcome resistance to IMiD treatment in MM cells.

IMiDs can specifically bind to cereblon, an important modulator of an E3 ubiquitin ligase complex, and alter the specificity of the substrates to be ubiquitinated (6). The changes in this specificity lead to the degradation of several transcriptional factors involved in tumor cell survival, such as IKZF1, IKZF3, and IRF4, in MM. Our results showed that a reduced dose of PD treatment did not induce cell death, as shown by the reduced induction of apoptosis and lower activation of caspase, even though prominent downregulation of IKZF1 and IKZF3 expression was observed. Hence, the downregulation of IKZF1 and IKZF3 was considered as an early event that is easily triggered during the action of IMiDs treatment. Although this suppression did not occur after treatment with a suboptimal dose of AFU, when combined with the PD treatment, the expression of IKZF1 and IKZF3 was predominantly suppressed. The precise mechanism of this enhanced suppression was not clear. Recently, Liu et al (28) proposed that kinase inhibition leads to a novel degradation process of IKZF1, instead of through the ubiquitin-proteasome pathway, in MM cells. In the current study, the additional administration of AFU and the subsequent pro-apoptotic activity, such as dephosphorylation of FOXO1, may support the PD-induced downregulation of IKZF1 and IKZF3 expression in a manner independent of cereblon-induced degradation. The mechanism by which the dephosphorylation of FOXO1 is correlated with the suppression of IKZF1 and IKZF3 expression is unclear. Recently, Alkhatib et al demonstrated the essential role of FOXO1 in appropriate mRNA splicing of IKZF1, which contributes to the stable expression of IKZF1, for the somatic rearrangement of immunoglobulin genes during B cell development (23). In MM cells, the effect of FOXO1 on the expression of IKZF1 is unclear. In reference to this previous report, the further study of how FOXO1 affects the expression of IKZF1 is warranted to explain the above concerns in MM cells. 
In conclusion, the AFU-PD combination therapy with suboptimal doses of PD and AFU exhibited remarkable anti-tumor activity in MM cells in comparison with the individual monotherapies. The mechanism of action of this combination therapy was dependent on the individual activities of PD and AFU without any interference with each other. The two subsequent actions, eIF4E inactivation caused by 4EBP1 dephosphorylation, and the enhanced suppression of IKZF1 and IKZF3, might induce additional effects through the AFU-PD combination therapy. The additional treatment of AFU with IMiD-based therapy should enhance the anti-tumor activity of IMiDs and overcome the resistance of IMiDs treatment. This combination therapy will exhibit more potent anti-tumor activity and fewer side effects when used clinically. Our study has provided the foundation for a new treatment strategy against RRMM with IMiD insensitivity or resistance, and improved IMiD-based therapy for patients with an intolerance to IMiD toxicity.

\section{Acknowledgements}

The authors would like to thank Ms. Chiori Fukuyama for her technical assistance. The study received financial support from Celgene Co., Ltd. This study was partly supported by a Grant-in-Aids for Scientific Research from the Ministry of Education, Culture, Sports, Science and Technology (nos. 16K07179 and 16K09855), National Cancer Center Research and Development Fund (no. 26-A-4), and the Practical Research for Innovative Cancer Control from Japan Agency for Medical Research and development, AMED (no. 15ck0106077h0002).

\section{References}

1. Quach H, Ritchie D, Stewart AK, Neeson P, Harrison S, Smyth MJ and Prince HM: Mechanism of action of immunomodulatory drugs (IMiDS) in multiple myeloma. Leukemia 24: 22-32, 2010.

2. Lonial S, Dimopoulos M, Palumbo A, White D, Grosicki S, Spicka I, Walter-Croneck A, Moreau P, Mateos MV, Magen $\mathrm{H}$, et al: Elotuzumab therapy for relapsed or refractory multiple myeloma. N Eng J Med 373: 621-631, 2015.

3. Dimopoulos MA, Oriol A, Nahi H, San-Miguel J, Bahlis NJ, Usmani SZ, Rabin N, Orlowski RZ, Komarnicki M, Suzuki K, et al: Daratumumab, lenalidomide and dexamethasone for multiple myeloma. N Eng J Med 375: 1319-1331, 2016.

4. Lonial S, Durie B, Palumbo A and San-Miguel J: Monoclonal antibodies in the treatment of multiple myeloma: Current status and future perspectives. Leukemia 30: 526-535, 2016.

5. Ito T, Ando H, Suzuki T, Ogura T, Hotta K, Imamura Y, Yamaguchi $\mathrm{Y}$ and Handa $\mathrm{H}$ : Identification of a primary target of thalidomide teratogenicity. Science 327: 1345-1350, 2010.

6. Lopez-Girona A, Mendy D, Ito T, Miller K, Gandhi AK, Kang J, Karasawa S, Carmel G, Jackson P, Abbasian M, et al: Cereblon is a direct protein target for immunomodulatory and antiproliferative activities of lenalidomide and pomalidomide. Leukemia 26: 2326-2335, 2012.

7. Fischer ES, Bohm K, Lydeard JR, Yang H, Stadler MB, Cavadini S, Nagel J, Serluca F, Acker V, Lingaraju GM, et al: Structure of the DDB1-CRBN E3 ubiquitin ligase in complex with thalidomide. Nature 512: 49-53, 2014.

8. Kronke J, Udeshi ND, Narla A, Grauman P, Hurst SN, McConkey M, Svinkina T, Heckl D, Comer E, Li X, et al: Lenalidomide causes selective degradation of IKZF1 and IKZF3 in multiple myeloma cells. Science 343: 301-305, 2014.

9. Chakraborty R, Muchtar E, Kumar S, Buadi FK, Dingli D, Dispenzieri A, Hayman SR, Hogan WJ, Kapoor P, Lacy MQ, et al: The impact of induction regimen on transplant outcome in newly diagnosed multiple myeloma in the era of novel agents. Bone Marrow Transplant 52: 34-40, 2017.
10. Durie BG, Hoering A, Abidi MH, Rajkumar SV, Epstein J, Kahanic SP, Thakuri M, Reu F, Reynolds CM, Sexton R, et al: Bortezomib with lenalidomide and dexamethasone versus lenalidomide and dexamethasone alone in patients with newly diagnosed myeloma without intent for immediate autologous stem-cell transplant (SWOG S0777): A randomised, open-label, phase 3 trial. Lancet 389: 519-527, 2017.

11. Shi CX, Kortum KM, Zhu YX, Jedlowski P, Bruins L, Braggio E and Stewart AK: Proteasome inhibitors block Ikaros degradation by lenalidomide in multiple myeloma. Haematologica 100: e315-e317, 2015.

12. Chapman MA, Lawrence MS, Keats JJ, Cibulskis K, Sougnez C, Schinzel AC, Harview CL, Brunet JP, Ahmann GJ, Adli M, et al: Initial genome sequencing and analysis of multiple myeloma. Nature 471: 467-472, 2011.

13. Spencer A, Yoon SS, Harrison SJ, Morris SR, Smith DA, Brigandi RA, Gauvin J, Kumar R, Opalinska JB and Chen C: The novel AKT inhibitor afuresertib shows favorable safety, pharmacokinetics and clinical activity in multiple myeloma. Blood 124: 2190-2195, 2014.

14. Narita T, Ri M, Masaki A, Mori F, Ito A, Kusumoto S, Ishida T, Komatsu $\mathrm{H}$ and Iida $\mathrm{S}$ : Lower expression of activating transcription factors 3 and 4 correlates with shorter progression-free survival in multiple myeloma patients receiving bortezomib plus dexamethasone therapy. Blood Cancer J 5: e373, 2015.

15. Ri M, Tashiro E, Oikawa D, Shinjo S, Tokuda M, Yokouchi Y, Narita T, Masaki A, Ito A, Ding J, et al: Identification of Toyocamycin, an agent cytotoxic for multiple myeloma cells, as a potent inhibitor of ER stress-induced XBP1 mRNA splicing. Blood Cancer J 2: e79, 2012.

16. Dumble M, Crouthamel MC, Zhang SY, Schaber M, Levy D, Robell K, Liu Q, Figueroa DJ, Minthorn EA, Seefeld MA, et al: Discovery of novel AKT inhibitors with enhanced anti-tumor effects in combination with the MEK inhibitor. PLoS One 9: e100880, 2014.

17. Munugalavadla V, Mariathasan S, Slaga D, Du C, Berry L, Del Rosario G, Yan Y, Boe M, Sun L, Friedman LS, et al: The PI3K inhibitor GDC-0941 combines with existing clinical regimens for superior activity in multiple myeloma. Oncogene 33: 316-325, 2014.

18. Li S, Pal R, Monaghan SA, Schafer P, Ouyang H, Mapara M, Galson DL and Lentzsch S: IMiD immunomodulatory compounds block $\mathrm{C} / \mathrm{EBP}\{$ beta\} translation through eIF4E down-regulation resulting in inhibition of MM. Blood 117: 5157-5165, 2011.

19. Han EK, Leverson JD, McGonigal T, Shah OJ, Woods KW, Hunter T, Giranda VL and Luo Y: Akt inhibitor A-443654 induces rapid Akt Ser-473 phosphorylation independent of mTORC1 inhibition. Oncogene 26: 5655-5661, 2007.

20. Rhodes N, Heerding DA, Duckett DR, Eberwein DJ, Knick VB, Lansing TJ, McConnell RT, Gilmer TM, Zhang SY, Robell K, et al: Characterization of an Akt kinase inhibitor with potent pharmacodynamic and antitumor activity. Cancer Res 68: 2366-2374, 2008.

21. Yamaji M, Ota A, Wahiduzzaman M, Karnan S, Hyodo T, Konishi H, Tsuzuki S, Hosokawa Y and Haniuda M: Novel ATP-competitive Akt inhibitor afuresertib suppresses the proliferation of malignant pleural mesothelioma cells. Cancer Med 6: 2646-2659, 2017.

22. Richter JD and Sonenberg N: Regulation of cap-dependent translation by eIF4E inhibitory proteins. Nature 433: 477-480, 2005.

23. Alkhatib A, Werner M, Hug E, Herzog S, Eschbach C, Faraidun H, Köhler F, Wossning T and Jumaa H: FoxO1 induces Ikaros splicing to promote immunoglobulin gene recombination. J Exp Med 209: 395-406, 2012.

24. Zhang X, Tang N, Hadden TJ and Rishi AK: Akt, FoxO and regulation of apoptosis. Biochim Biophys Acta 1813: 1978-1986, 2011.

25. Fu $\mathrm{Z}$ and Tindall DJ: FOXOs, cancer and regulation of apoptosis. Oncogene 27: 2312-2319, 2008.

26. Greer EL and Brunet A: FOXO transcription factors at the interface between longevity and tumor suppression. Oncogene 24: 7410-7425, 2005.

27. Raje N, Kumar S, Hideshima T, Ishitsuka K, Chauhan D, Mitsiades C,Podar K, Le Gouill S, Richardson P, Munshi NC, et al: Combination of the mTOR inhibitor rapamycin and CC-5013 has synergistic activity in multiple myeloma. Blood 104: 4188-4193, 2004.

28. Liu Y, He X, Sui Y, Yu R and Xu G: Transcription factor IKZF1 is degraded during the apoptosis of multiple myeloma cells induced by kinase inhibition. FEBS Lett 589: 2233-2240, 2015. 